\title{
Prospective Usage of the Internet of Bio-Nano Things For Very Anticipated Detection of Proteins in Type-2 Diabetes Patients: Stopping the Progress of Diabetic Nephropathy with Nanomedicine
}

\author{
Huber Nieto-Chaupis \\ Universidad de Ciencias y Humanidades, \\ Center of Research eHealth \\ Av. Universitaria 5175, Los Olivos Lima39 Perú \\ huber.nieto@gmail.com.
}

\begin{abstract}
A natural upgrade of the Internet of Things (IoT) and the Internet of Nano-Things (IoNT) is the so-called Internet of Bio-Nano Things by the which the organs of the human body would be part of a communication's network and emitting signals continuously. In this paper we have paid attention to the prospective scheme of surveillance that aims to measure the charged bunches of proteins which are fluxing through the renal glomerulus. We propose the idea that these bunches of albumin might have anomalous displacements out the glomerulus due to the presence of dipoles of glucose. Our theoretical formulation consists essentially in a physics model. Under this view, the electrodynamics of albumin is governed by repulsive and attractive forces that pushes out theses proteins to the spaces of urine formation. For this end, we apply electrodynamics to calculate the electric potential and fields. All these processes might be seen as the very beginning of the well-known diabetes kidney disease. Under the assumption that the presence of a nanodevice contains capabilities for sensing the bunches of albumin, we estimated the efficiencies of charge detection using the Monte Carlo method. We present simulations about the possible tracks of albumin after of their interaction with others charged negatively cells like podocytes. The present analysis has considered others charged proteins which would play the role of noise or background. This methodology is embedded in the framework of the IoBNT and the respective quality of service is estimated as well.
\end{abstract}

Index Terms-IoBNT, type-2 diabetes, diabetes kidney disease, quality of service.

\section{INTRODUCTION}

$\mathbf{W}$ ITH With the advent of the prospective applicability of a multi-purpose nanotechnology is expected that it achieves a notable role in the territory of human medicine and provide novel tools to reduce progressively the global burden of diseases. [1][2][3][4][5][6]. For instance it's not difficult to perceive that among the main causes of mortality in the world is due to cancer and probably diabetes (type 1 and 2) [7][8]. In order to face the different levels of disease's complications, the synergy of various disciplines is required. For instance, the conjunction of the capabilities of the nanotechnology and techniques of the classical medicine is often called nanomedicine by which is expected to tackle the progress of disease and, in this manner the reduction of mortality might be realistically foreseen [9]. Clearly, advanced surveillance systems and multidisciplinary schemes will be required [10][11][12]. One of the main role of nanomedicine will be the identification of anomalous and dysfunctional evolution of organs in time. The identification might be inspired on traditional techniques of the engineering such as the sensing or detection of signals. The output of these processes might be included in communication systems such as the Internet [13][14][15][16][17]. In this manner, the microscopic events which are under continuous surveillance by cyberhuman interfaces would upgrade the Internet networking to one which is recently called Internet of Bio-Nano Things (IoBNT). In effect, the IoBNT aims to include the continuous monitoring of bio-chemical signals in the molecular level such as the cell communication done by the electrolytes like $\mathrm{Ca}^{2+}$, for instance. Anionic or cationic signals would constitute a type of advantage for the nanomedicine, since one expects that the software of nanodevices might consist in algorithms based on concepts of physics of charged objects. A topic of interest that groups all these facts is in the side of the expected complications of the type-2 diabetes, in particular the so-called diabetes kidney disease (DKD) which has turned out to be as one of the worst complications of diabetes in patients. Most of the behavior of kidney is due to the properties of electric charge in the last layers previous to the urine formation [17]. A crucial process involving the dynamics of electric charges in kidney takes place in the renal glomerulus whose essential task is that of stopping of the abnormal flux of charged proteins like albumin to the urine. These facts are well treated in a full scenario of electrodynamics which helps to understand and apply a formalism based on attraction and repulsion forces. From the point-of-view of nephrologist the urine albumin excretion (UAE) is an important indicator of how the kidney is under continue degradation due to diverse factors, being the most important the high concentrations of glucose in blood. In this paper, we discuss the prospective applicability of the IoBNT as a non-conventional methodology to anticipate the progressive damage of the renal glomerulus due to negatively charged proteins as albumin, in those type- 2 diabetes patients. 
We sustain this argument through fundamental physics which is based on the idea that the dipole moment of glucose is the main cause of negative charge depletion in the renal glomerulus. Consequently, the bunches of albumin takes advantage of this opportunity to surpass spaces reaching the Bowman's space where the formation of urine takes place. We explore the possibility that the effective sensing of charged proteins might be achieved by a nanosensor which is expected to be deployed a few nanometers from the renal glomerulus. We assume that this nanodevice would have to have electric properties in order of interacting with the charged proteins. The electric interaction is translated in terms of electric oscillations in where the repulsive forces between same sign electric objects would produce damped oscillations in the plane of interaction. A first approximation of this considers that the nanodevice and bunches of negative charges are in the same plane or are coplanar each other. In this manner, the dynamics that exhibits the nanodevice due to the electric interactions with albumin continuously, is used as input signal to produce the correspond output: THz waves. In fact, inside the IoBNT the continue emission of $\mathrm{THz}$ pulses by the nanodevice might represent a novel technique of in situ surveillance only if the pulses have been detected by bio-cyber interface already working inside a IoBNT networking. We expect that the networking is storing the acquired data which should be read by the doctor or nephrologist. Depending of the flux of albumin per unit of time, the specialist would take rapid intervention. Nowadays, type-2 diabetes are reporting the kidney disease after 5 or more years from the beginning of disease. In most cases, patients are passing last phases and become candidates for dialysis processes.

The rest of this paper is structured as follows: In second section, the case of the renal disease and DKD is presented. Furthermore, we present a model of deterioration of podocytes. Third section aims to explain the physic model of such interaction. In order to be consistent with the main argument of charge interaction, we use physics equations based on electricity. Once the Laplace's equation is solved, the electric fields and forces are derived. The usage of the Coulomb's force is justified since it can explains the repulsion and subsequent displacement of negative charges located in the glomerulus and podocytes. Our main assumption is the existence of attractive and repulsion electric forces which might be responsible of the dynamical cancellation of the shield of charges in the glomerulus. This idea might explain the why of the anomalous flux of proteins like albumin through the urine. All these equations become the basis of the algorithm for simulation of the dynamic of the charges. In fourth part, we build a model for sensing the albumin by using the well-known methodologies of input and output. In this manner, we model the electric properties of the glucose and glomerulus, that are the main ingredients to calculate the full output of system. We have included noise in the formalism, since the presence of others proteins will notably affect the detection of albumin. For instance, the Tamm Horsfall proteins would represent the main source of background for the signal. Here, also we perform a estimation of the expected values of albumin in typical cases and compare to the known clinical results. A Monte

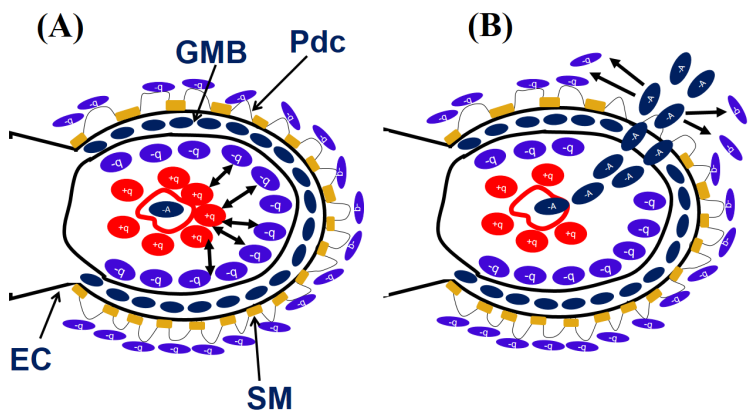

Fig. 1. Sketch of the main idea of paper: (A) Attractive forces between the positive charges of glucose's dipole and negative charges along the epithelial cells (EC) and glomerular membrane basement (GMB). (B) After the charge depletion, bunches of albumin reaches and crosses the slit membrane (SM) exerting repulsion forces on the remaining charges located in the surface of podocytes. Finally, albumin would reach the space where urine is formed.

Carlo simulation of the processes containing the physics of the charges in the external spaces of glomerulus in the fifth section is given. Essentially, we have simulated the detection of the charges and measured the damage in podocytes as consequence of excess of negative charges passing along the glomerulus. Once the nanosensor is deployed nearly to the kidney, few nanometers from the glomerulus, the surveillance of the quantity of albumin excreted into the urine would be unstoppable. In sixth section, a crude estimation the qualityof-service $(\mathrm{QoS})$ of the network is presented. The network is assumed to be running inside of the IoBNT framework. The emission of pulses by the nanosensor in the band of $\mathrm{THz}$ is received by bio-interfaces which are connected to cloud. The access to these data can be done by doctor or nephrologist whom can assess and evaluate the state of the layers of kidney remotely. It might open new ways to apply or reconfigure the pharmacology to tackle down the dysfunctional evolution of kidney. The estimated efficiencies of previous section for albumin detection enters as input in this part. The absorption of waves by water inside the human body is stressed. This intrinsic trouble might reduce the signal efficiency in the side of the bio-interface device. Finally, the conclusion of the paper is presented.

\section{Possible Origin of Renal Damage in TyPe-2 Diabetes Patients}

According to clinic data, diabetic patients are strong candidates to acquire DKD which is consensually considered as one of the latest phases during the progress of diabetes along the time. In Fig. 1, left and right panels, would explain the very beginning of the renal damage. Since higher concentrations of glucose in blood (normal glucose's range lies between 70 $\mathrm{mg} / \mathrm{dl}$ and $110 \mathrm{mg} / \mathrm{dL}$ ) would be a reason of the presence of irreversible complications such as diabetic retinopathy and necrosis, it also might be the cause for the beginning of the degradation of the renal function. In left panel, the form how the electric property of the albumin and glucose makes changes in the glomerular layers is sketched. While the abundance of glucose is expected, in same proportion the albumin proteins would "escape" of their containers or microvessels. In 


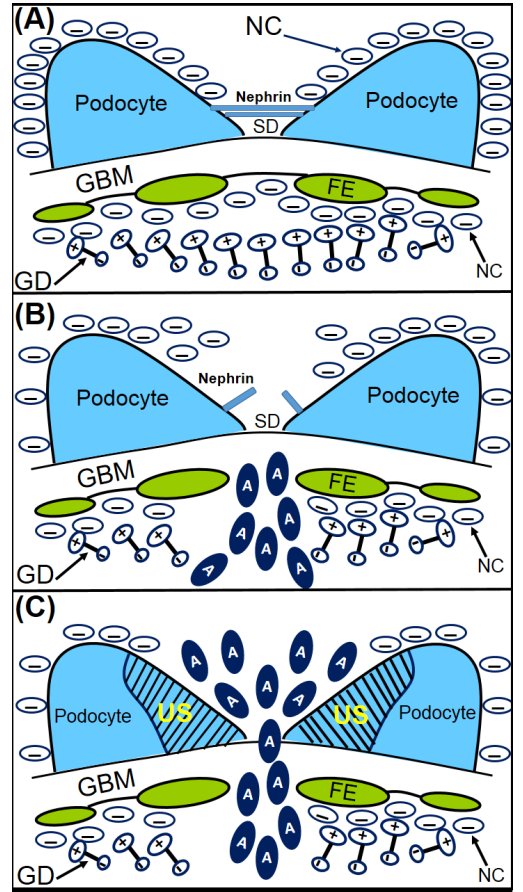

Fig. 2. Sketch of the deterioration of podocytes due to electric effects. (A) Normal podocytes contains negative charges over their surfaces and exchanging communication with others through Nephrine. (B) The reduction of charges on the epithelium is translated as the pass of albumin through the GBM. It would affect the functionality of mediators like the Nephrin. (C) When albumin is passing through the SD depletes and moves away the remaining charges of podocytes, creating unprotected surfaces and initializing the deformation of their morphologies.

high concentrations, these bunches of albumin are trespassing the glomerular membrane basement and the epithelial cells. In right panel the case where albumin (and others proteins) are leaving the glomerular layers and reaching and modifying the surfaces where podocytes are located.

One of the most relevant characteristics of DKD is the absence of pain in the urinary channels for several years, despite of the fact that the DKD has already started. DKD is strongly related to the cases of albuminuria, whose numerical understanding is as follows

$$
\mathcal{M}=\left\{\begin{array}{lr}
\text { normal } & 0<\mathcal{W} \leq 30 \mathrm{mg} / \mathrm{dL} \\
\text { microalbumin } & 30<\mathcal{W} \leq 300 \mathrm{mg} / \mathrm{dL} \\
\text { macroalbumin } & \mathcal{W}>300 \mathrm{mg} / \mathrm{dL} .
\end{array}\right.
$$

The quantity of albumin detected in lab test is often measured as UAE ( $\mu \mathrm{g} / \mathrm{min})$. High values of UAE might be imminently correlated to the beginning of the renal failure.

\section{A. Deterioration of Podocytes}

In the Fig. 2, the proposed scenario where renal glomerulus is damaged by effect of higher concentrations of glucose in diabetes patients's blood is sketched. In (A) the case of a stable glomerulus, where blood vessels are transporting plasma, proteins, glucose, and other compounds is depicted. This figure is explained as follows: high concentrations of glucose in vessel bloods would imply the presence of bunches of positive charges because the dipole moment. The positive charges produce attraction's electric forces with the negative charges that involves the glomerulus membrane basement (GMB) and located along the fenestrated endothelium (FE) which is normally protected by a shielding of negative charges whose role is that of stopping the pass of giant proteins like albumin. The increasing of glucose concentrations would vary the homeostasis of the electric surface of endothelium. It is because the glucose dipole (GD) glucose would induce to cancel the negative charges. In this manner, the only attractive electric forces would be starting with the depletion over the FE. For this view, we have assumed a curved morphology in according to Fig. 1. In (B) under the assumption that the albumin as molecule has a ellipsoid-like shape of $3.8 \mathrm{~nm}$ in diameter and $15 \mathrm{~nm}$ long, and the the slit diaphragm (SD) is or order of 5 a $10 \mathrm{~nm}$, one expects that these giant proteins have enough space to be filtrated [18]. In effect, once the negative charges are being depleted the renal filtration of macromolecules might be initialized. It would be the basis of the well-known molecular mechanisms of hyperfiltration. Neutral porosity of the glomerular walls turns out to be favorable to the pass of bunches of albumin. When albumin reaches and breaks the SD these proteins exert repulsion forces with the remaining negative charges located on the surfaces of podocytes. The the group of albumin with negative charge produces dysfunctional effects on the nephrine, and the new charge configuration makes unprotected the electric surface of podocytes. Since communication between adjacent podocytes is lost, remaining charges are distributed asymmetrically on the podocytes surface. It is because the repulsive electric force between albumin and the remaining ones located on the podocytes would produce a new relocation of charges which are not protecting the glomerulus anymore. In (C) the situation is getting to be dramatic because albumin crosses the SD together to other proteins and moves through the Bowman's space. This scenario would indicate the first moment of the glomerular damage, where podocytes are already lost an important area of the shielding of negative charges. This area is called unprotected surface (US). In this manner, it is imminent that the podocytes experience a substantial deformation in their morphologies just as it is seen in lab tests. Here, one expects that the electric dynamics is entirely governed by repulsive forces. The evolution in time of these processes might be the reason to argument the deterioration of the morphology of the podocytes due to electric effects more than biochemistry reactions. Furthermore the pass of negatively charged albumin would move away the remaining charges from their initial positions making much weak the protection of the podocytes area. The filtered proteins become part of the processes of urine formation through the convoluted tubule.

\section{Physics of the Glomerular Damage}

\section{A. Electrodynamics in the Glomerulus's Area}

According to the possible configuration of renal damage presented in Fig. 2, the pass of albumin through the urine would have its origin in electric processes purely. Normally, clinic tests have been using the UAE as unit of measurement without to include concepts of electric charge. The UAE is 

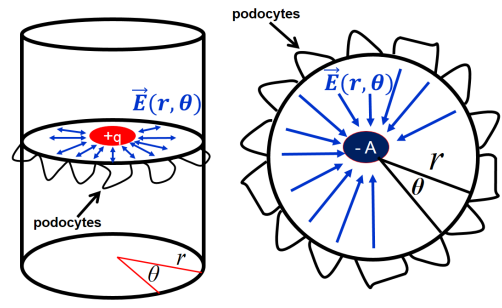

Fig. 3. Left: Geometrical configuration of the renal glomerulus where the center indicates the presence of positive charge due to the glucose dipole moment. Right: Field lines due to the presence of the negatively charged albumin exerting repulsive forces on the charges of the GMB and podocytes seen in a coplanar geometry or two-dimensional space.

expressed in terms of mass per unit of time. In the following, we attempt to relate the UAE with physics concepts based on electric fields. Under the assumption that the rate of excretion is proportional to the electric force between the bunches of albumin and negative charges belonging to the regions of GMB and podocytes, we propose the following definition $\vec{F}=q \vec{E}$ $=\frac{\operatorname{mass}}{\text { time }} \vec{v}=[\mathrm{UAE}] \vec{v}$, for the cases where $\vec{v}$ (the velocity of the albumin bunches passing through the slit membrane) is constant, therefore we can arrive to the following relation

$$
[\mathrm{UAE}]=\frac{|q \vec{E}|}{|\vec{v}|}=\frac{|q \vec{\nabla} \Phi|}{|\vec{v}|}=\beta \vec{\nabla} \Phi
$$

where $\beta=\frac{|q|}{|\vec{v}|}$ and $\Phi$ the electric potential that satisfies the Poisson's equation $\nabla^{2} \Phi=-\frac{\rho}{\epsilon}$, and the charge density obeys $\vec{\nabla} \cdot \vec{E}=\frac{\rho}{\epsilon}$ the well-known Gauss's law.

\section{B. Estimation of the Electric Field and Forces in the Podocytes Area}

In Fig. 3, the possible geometrical configuration which is suitable to apply physics equation considering the area of podocytes and albumin filtration, is sketched. Thus is plausible to accept that the morphology glomerular fits to some extent the cylindrical coordinates system (left panel). Firstly, we face the problem of calculating the electric fields when wehave two variables: radial and angular (right panel). The solution of the Poisson's equation becomes more manageable by using the method of Green's functions. Thus, we can write the following solution [19],

$$
\Phi(r, \theta)=\sum_{n}^{\infty}\left[\mathcal{C}_{n} r^{n} \operatorname{Sin}(n \theta)+\mathcal{D}_{n} r^{-n} \operatorname{Cos}(n \theta)\right] .
$$

We see that the electric potential depends on the constants $\mathcal{C}_{n}$ and $\mathcal{D}_{n}$ while $n$ integer number running for all values. This solution conciliates well the standard procedures for solving the Poisson's equation by which the variables separation's method is used. From Eq. (3) the corresponding electric field can be exactly calculated through $\vec{E}=-\vec{\nabla} \Phi(r, \theta)$ resulting as

$$
\begin{aligned}
& \vec{E}_{r}=\sum_{n}^{\infty}\left[\frac{\mathcal{D}_{n} \operatorname{Cos}(n \theta)}{r^{2}}-\mathcal{C}_{n} \operatorname{Sin}(n \theta)\right] \hat{e}_{r} \\
& \vec{E}_{\theta}=\sum_{n}^{\infty}\left[-\frac{\left.\mathcal{C}_{n} r \operatorname{Cos}(n \theta)-\frac{\mathcal{D}_{n} \operatorname{Sin}(n \theta)}{r}\right]}{r}\right] \hat{e}_{\theta} .
\end{aligned}
$$

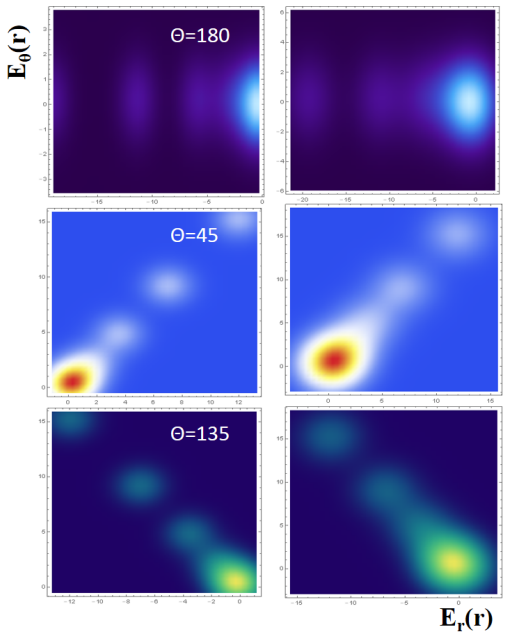

Fig. 4. Possible configurations of electric field's components depending on the radial variable but fixed for three different angles. Top panels describe the cases when $\theta=180$ degrees. Middle plots display the scenarios where $\theta=45$ degrees. In bottom panels are shown the scenarios for $\theta=135$ degrees.

When $r \rightarrow \infty$, the only contribution is the radial part, which is given by the term $\vec{E}_{r} \approx \mathcal{C}_{n} \operatorname{Sin}(n \theta) \hat{e}_{r}$. From the qualitative view, one expects that the electric force is governed by sinusoidal shapes. This gives us advantages to build arguments about the forms of field in the areas and spaces around the glomerulus.

\section{Generalization of the Electric Potential}

Conserving the full cylindric geometry as shown in left panel of Fig. 3, with a radius $a$ and length $L$, the electric potential calculated in $\overrightarrow{\mathbf{r}}$ from a bunch of albumin molecules whose net charge is denoted by $\mathcal{Q}$ located in $\overrightarrow{\mathbf{r}}^{\prime}$ can be written as [20],

$$
\begin{aligned}
& \Phi\left(\overrightarrow{\mathbf{r}}, \overrightarrow{\mathbf{r}}^{\prime}\right)=\frac{2 \mathcal{Q}}{\pi \epsilon L a^{2}} \sum_{\ell, m, n} \frac{\operatorname{Exp}\left[i m\left(\theta-\theta^{\prime}\right)\right]}{\left[\left(\frac{\xi_{m, n}}{a}\right)^{2}+\left(\frac{\ell \pi}{L}\right)^{2}\right] J_{m+1}^{2}\left(\xi_{m, n}\right)} \times \\
& \times \operatorname{Sin}\left(\frac{\ell \pi z}{L}\right) \operatorname{Sin}\left(\frac{\ell \pi z^{\prime}}{L}\right) J_{m}\left(\frac{\xi_{m, n} r}{a}\right) J_{m}\left(\frac{\xi_{m, n} r^{\prime}}{a}\right)
\end{aligned}
$$

where $\epsilon$ the electric permittivity and is related to dielectric constant with a value equivalent to water, which is lying in the rage of 1 up to 100 [21]. In praxis, a suitable choice is the value of 80 , same as water. The modes $\xi_{m, n}$ are depending on the integers $m$ and $n$ as consequence of the usage of the variables separation. The $J_{m}$ are called integer-order Bessel functions and essentially depend on the radial variable. Clearly, in the cases where $\left(\frac{\ell \pi}{L}\right)^{2}>>\left(\frac{\xi_{m, n}}{a}\right)^{2}$ and $\frac{\ell \pi}{L} \rightarrow \infty, \Phi \rightarrow 0$. While we more move out from glomerulus area, the fields intensity are getting weak. Thus we can attach an exponential part $\operatorname{Exp}(-\beta t)$ (with $\beta$ a real parameter) for which it becomes negligible for large times in congruence with the radial part. In addition, when only the radial variable is kept, the potential and field electric are fully depending on the Bessel function.

$16^{\text {th }}$ LACCEI International Multi-Conference for Engineering, Education, and Technology: "Innovation in Education and Inclusion”, 19-21 July 2018, Lima, Peru. 


\section{Angular Analysis of Bunches}

In Fig. 4, various simulated scenarios obtained with [22] are shown. Basically, we try to obtain a general picture of the possible cases where charged bunches of albumin are "escaping" from the glomerulus in agreement to the direction of the fields. Since the model does not include variations in time, the plots are indicating the possibles static morphologies up to three different angular positions. Actually the knowledge of the shapes of the bunches of albumin would serve to make calibrations and hardware configurations in the nanodevice. For instance, the tracks exhibited by the albumin when is leaving the glomerulus [23][24][25][26][27][28][29][30][31][32] would be of crucial importance for the nanosensor. The present analysis has considered the plane $E_{\theta}(r)$ vs. $E_{r}(r)$ for three angles: $\theta=45,135$, and 180 degrees. We used Eq. (4) and (5) with $n$ running from -5 to 5 . Others orders have not substantial effect. The values of $\mathcal{C}_{n}$ and $\mathcal{D}_{n}$ were estimated to be of 0.001 in average. The plotting used the technique of bivariate bandwidths (bw) expressed in units of standard deviation. For all panels, left and right column: 1.20 and $2.00 \mathrm{bw}$, respectively. Top panels: the case where $\theta=180$ degrees. This case would correspond to the scenario where the tangential component of the electric field moves the charges of podocytes to the left side off. In middle panels are the scenarios where the electric field is fully radial for $\theta=45$ degrees. This is the best scenario by which the albumin proteins are move out forward on the convoluted tubule. For bw $=2.0$ (right panel) shows that the sinusoidal character of the electric potential dominates the shape of the electric field. Finally, in bottom panels are the scenarios where $\theta=135$ degrees. Again, the coloration seen in the plots would indicate that the electric field is strongly dominated by the radial part. For bw=2.0 (right panel) is seen that the field is homogeneous since the points around $(0,0)$ is uniform, a bit different from the one seen in middle plots. A straightforward calculation of the velocities derived from the radial component considers $v=\sqrt{\frac{q E_{r}}{m_{B}}} \approx 10^{-4} \mathrm{~m} / \mathrm{s}$, where $m_{B}$ the effective mass of charged bunch. All these simulations are understood as a kind of advantage in the side of the nanosensor aiming a good signal reception. Once the nanosensor is implemented and located nearly to the glomerulus, the main task would be that of sensing the electric oscillation caused by the repulsive forces caused by the charged albumin.

\section{Monte Carlo Simulation for Experimental DETECTION}

\section{A. Deployment of Nanosensor}

The prospective nanodevice has the role the sensing of negatively charged bunches which are surpassing the depleted surfaces of the podocytes. Hypothetically, the sensing of large bunches of albumin might be a kind of indirect measurement of damage in the morphology and functionality of last layers of the glomerulus. Since both nanodevice and albumin would have same sign, one expects that the repulsion force might produce dynamical oscillations that would be a type of electric detection. In one hand, nanodevice is expected to be 1 to $2 \mathrm{~nm}$ away of last layers of glomerulus and radius of around or less
TABLE I

LIST OF RANDOM AND SEMI-RANDOM VARIABLES AND CONSTANTS (FROM EQ. 15).

\begin{tabular}{|c|c|c|c|}
\hline Variable & Range & Type & Related to \\
\hline$q$ & $0-5$ & random & albumin \\
\hline$j$ & $0-5$ & random & noise \\
\hline$a_{1}$ & $0-10$ & semi-random & albumin \\
\hline$a_{2}$ & $0-20$ & semi-random & noise \\
\hline$\lambda_{1}$ & $0-1$ & random & charge depletion in podocytes \\
\hline$\lambda_{2}$ & $0-1$ & random & others proteins \\
\hline
\end{tabular}

than $1 \mathrm{~nm}$. On the other hand, large bunches of albumin would have as radius a size approximately of $10 \mathrm{~nm}$, which is of order of the size of slit diaphragm aperture. Nanodevice would have to have negative charges over its surface. It is because, the repulsion forces exerted by the bunches of albumin will move oscillatory inside its center-of-mass. This mechanical movement is required to active hardware for emitting signals. Nanodevice is therefore designed to receive electric signals from albumin and send signals to a receptor (external interface) in the $\mathrm{THz}$ band as established in the proposed scheme of the IoBNT.

\section{B. Aleatory Selection of Random Variables and Calculation of Total Electric Force}

Because these processes are governed by physics and chemistry laws in the range of $\mathrm{nm}$, it is suitable to apply stochastic methods to obtain a rough estimate of the possibilities to detect signal and intrinsic background or others proteins and negatively charged wastes. Once the solution of Eq. 5 is known the we can calculate the electric forces normalized to unity,

$$
\mathcal{F}=-\vec{\nabla} \Phi(\vec{r}, t)
$$

Thus it is possible to rewrite the normalized total force as

$$
\begin{gathered}
\mathcal{F}_{T}\left(\omega_{1}, \omega_{2}\right)=-\lambda_{1} \int \mathcal{F}_{S}(\omega) \delta\left(\omega-\omega_{1}\right) d \omega \\
-\lambda_{2} \int \mathcal{F}_{B}(\omega) \delta\left(\omega-\omega_{2}\right) d \omega=\mathcal{F}_{S}\left(\omega_{1}\right)+\mathcal{F}_{B}\left(\omega_{2}\right) .
\end{gathered}
$$

where the $\lambda_{1,2}$ are the weights. In Table I, are described the random variables, indicating their range, type and relation to the specific processes. According to this the numbers $q, j$ and $\lambda_{1,2}$ are extracted from a numbers aleatory machine, whereas $a_{1,2}$ do not necessarily are random numbers. We target to plot the intensity of the electric force detected by the nanosensor coming from the albumin, preferentially. It should be noted that the albumin bunches might have a large size in comparison to others background compounds. So that, the highest intensities of electric force would come from the albumin, essentially. Minor intensities are defined as the background or noise. The algorithm 1 describes the computational methodology to calculate the total electric force. Line-1 defines the number of events. Because the order of the Bessel functions are taken as random numbers as listed in Table I, between lines 2 and 6 are defined the pairs $(q, j)$. Indeed, the quantities $a_{1}$ and $a_{2}$ are prepared in according to their ranges (lines 7 and 12). Between lines 13 and 26, 


\begin{tabular}{|c|}
\hline Algorithm 1: Monte Carlo Simulation \\
\hline $\begin{array}{ll}1 & \text { repeat for } \mathcal{N} \text { events } \\
2 & \text { for } q=1 \rightarrow \mathcal{Q} \text { do } \\
3 & \text { for } j=1 \rightarrow \mathcal{J} \text { do } \\
4 & \text { Save pair }(q, j) \\
5 & \text { end for } \\
6 & \text { end for } \\
7 & \text { for } k=1 \rightarrow \mathcal{K} \text { do } \\
8 & a_{1}=0.005 \\
9 & a_{2}=0.01 \\
10 & a_{1}(k)=a_{1}+0.005^{*} \mathrm{k} \\
11 & a_{2}(k)=a_{2}+0.01^{*} \mathrm{k} \\
12 & \text { end for } \\
13 & \text { for } p=1 \rightarrow \mathcal{P} \text { do } \\
14 & \text { call random }(q, j) \\
15 & \text { call } a_{1}\left(p_{1}\right) \\
16 & \text { call } a_{2}\left(p_{2}\right) \\
17 & \text { call random } \lambda_{1} \\
18 & \text { call random } \lambda_{2} \\
19 & \text { if } \lambda_{1} \neq \lambda_{2} \text { then } \\
20 & \text { for } \ell=1 \rightarrow \mathcal{L} \text { do } \\
21 & \omega_{1, \ell} \rightarrow \beta_{\ell} x_{1, \ell} \\
22 & \omega_{2, \ell} \rightarrow \beta_{\ell} x_{2, \ell} \\
23 & \mathcal{F} \mathcal{F}_{T}=\lambda_{1} \mathcal{F}_{S}\left(\beta_{\ell} x_{1, \ell}\right) \otimes \eta_{1, \ell}+\lambda_{2} \mathcal{F}_{B}\left(\beta_{\ell} x_{2, \ell}\right) \otimes \eta_{2, \ell} \\
24 & \text { if } \ell=\mathcal{L} \text { then } \\
25 & \Delta \mathcal{F}=\sqrt{\left(\frac{\Delta \mathcal{F}_{T}}{\Delta x_{1, \ell}} \Delta x_{1, \ell}\right)^{2}+\left(\frac{\Delta \mathcal{F}_{T}}{\Delta x_{2, \ell}} \Delta x_{2, \ell}\right)^{2}} \\
26 & \text { end if } \\
27 & \text { end for } \\
28 & \text { end if } \\
29 & \text { end for } \\
30 & \text { until } \mathcal{N} \rightarrow \mathcal{N}_{\mathrm{MAX}} \\
& \end{array}$ \\
\hline
\end{tabular}

the Monte Carlo step is achieved. Firstly, we extracted a pair $(q, j)$ in an aleatory manner, together to $a_{1}\left(p_{1}\right)$ and $a_{2}\left(p_{2}\right)$. Subsequently, we provide random values to $\lambda_{1,2}$. The Monte Carlo step is reflected in line 19, which is understood as the acceptance or rejection (or abort) of the process. When the acceptance is confirmed we proceed to calculate $\omega_{1, \ell}$ and $\omega_{2, \ell}$, which are proportional to the distances measured in $\mathrm{nm}$. All this gives us capabilities to calculate $\mathcal{F}_{T}$ (line 23) as a 3D plot. In praxis, we have projected the $3 \mathrm{D}$ surface to $2 \mathrm{D}$ as shown in Fig. 4, where the intensity of electric force appears as function of distances measured in $\mathrm{nm}$ (multiplied by a factor 10). Intrinsic errors associated to the numerical procedures are calculated in line 25. Actually, main source of errors would come of the definitions as written in lines 21 and 22, for each Monte Carlo loop. It should be noted that the variations or deviations in $\mathcal{F}_{T}$ appear also by the presence of the random functions $\eta_{1,2}$ which are expected to provide uncertainties to the numerical calculations. For the present case, $\mathcal{N}_{\text {MAX }}=133$.

\section{Results and Interpretation of Plots}

In Fig. 6. possible scenarios for sensing the bunches of proteins are displayed. In order to precise the interpretations of resulting simulations, we explain the frame or coordinates system used in the simulations. We define the radial distances as $x_{1,2}=\sqrt{\bar{x}_{1,2}^{2}+\bar{y}_{1,2}^{2}}$ where $\bar{x}$ and $\bar{y}$ the usual Cartesian
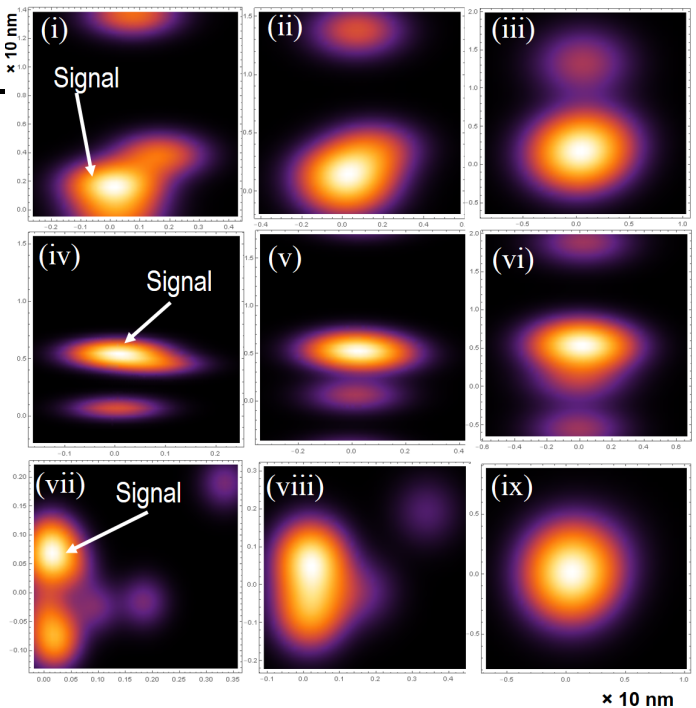

$z$
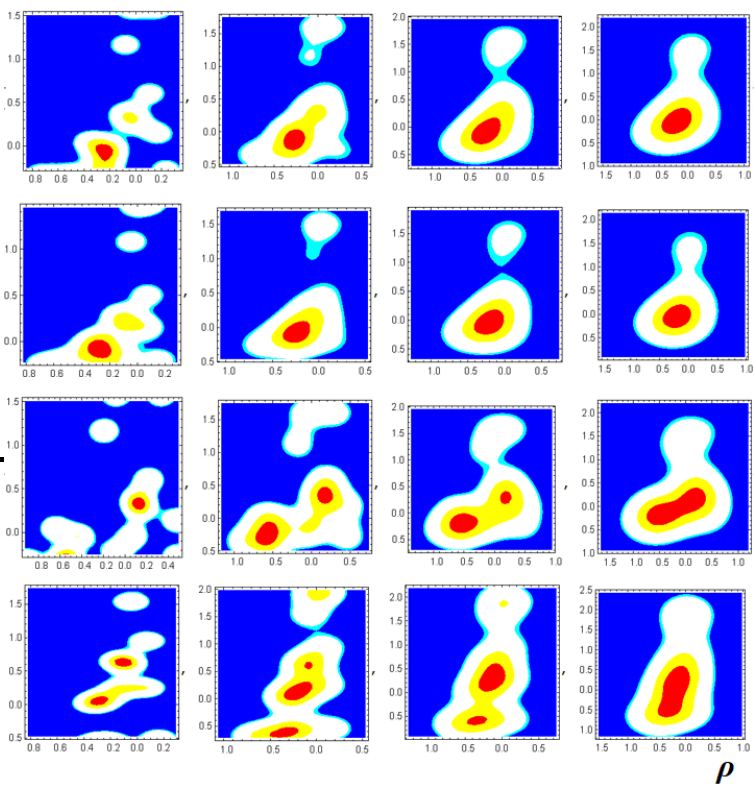

Fig. 5. Top plots: Various spatial configurations of the possible localizations of the signal (indicated by the white arrow) and noise (others negatively charged proteins). The plot (i), (ii) and (iii) show the cases where signal and noise abandon each together the last glomerular layers resulting in pessimistic scenarios. Contrarily, in (iv), (v) and (vi) might be the more favorable scenarios to detect albumin away from noise. The plot (iv) would denote the best scenarios where signal can be detect with an efficiency or order of $70 \%$. Bottom plots signal and noise assumed that both start to be indistinguishable each other plotted by using Eq. 5 conserving the radial and longitudinal variables

coordinates. All these quantities are incorporated in lines 21 and 22. Our assumption is that the last layer of glomerulus is located in the origin $(0,0)$. The objective of the simulation is to identify the position of the intensities of electric force which is analogue to detect bunches of albumin to optimize the localization of the sensor. In top left, is seen the case the case when signal (albumin) might be detected around of $1 \mathrm{~nm}$ away from the origin. The bunching of albumin here is indicated through the white arrow. Noise is easily distinguishable beyond $2 \mathrm{~nm}$. In the top center plot, we see the case 
where signal and noise are superimposed each other making indistinguishable the signal. In the upper part a minor presence of charged proteins is viewed. It is interpreted as a stochastic fluctuation of the numerical calculations and Monte Carlo. In top right plot, a third scenario is plotted. Here again, signal and noise are well together, resulting in a signal efficiency of order of $10 \%$. The middle left plot would represent a desired case. Here, signal and noise are well separated in around $1 \mathrm{~nm}$. We suggest that a possible position of the nanodevice would be approximately $\sqrt{26} \mathrm{~nm}+\delta \mathrm{nm}$ away from the glomerulus ( $\delta$ denotes the associated error). In this case the efficiency has turned out to be of order of $48 \%$ Middle center plot indicates the oncoming of noise to the signal. Same morphology is again encountered in the middle right plot where signal and noise are already forming one bunch of proteins. These last two scenarios would make difficult the sensing of albumin, and their efficiencies have been of order of 12 and $9 \%$, respectively. Finally, in bottom left plot, we observe the case when signal appears to keep a substantial separation from the origin in around $5 \mathrm{~nm}$. The negative value of the position of noise is interpreted as the case where only albumin might be detected in such distance, whereas part noise is still fluxing through the podocytes. Middle center and right plots are showing the scenario where signal and noise flux out the glomerulus already attained each other at the beginning of the convoluted tubule. In average, one third of the presented plots shows seen to be favorable, in the sense that the signal can be detected with an efficiency above $70 \%$, which is $15 \%$. In bottom panel of Fig. 6 we see as signal and noise are both superimposed by avoiding to distinguish each other. Clearly this fact might also be interpreted as an advanced level of degradation of the podocytes [33][34][35][36][37][38][39][40]. It also would indicate that the role of the nanodevice would be limited to the cases where the glomerulus has started to be electrically damaged as effect of the presence of large concentrations of glucose in blood. This information brought to a network of IoBNT would be beneficial to the patients since they might know in advance the progress of the disease, in particular the loss of the electric shielding of the glomerulus which is crucial information to avoid the flux of albumin in urine [41][42][43][44]. Finally, the Monte Carlo error was estimated to be $\Delta \mathcal{F}_{T}$ of order of $12 \%$.

\section{Elements of the IoBNT Network Applied to NANOMEDICINE}

In Fig. 6, the elements corresponding to the case where a type-2 diabetes patient is part of the IoBNT network [45][46][47][48][49][50], is sketched. A nano device carries out the action of sensing the flux of charged proteins through the convoluted tubule where the urine is formed. This nano device is located a few nanometers of the renal glomerulus. The detection of albumin is synonymous of $\mathrm{THz}$ waves radiation in an isotropically manner. The reception of this radiation is achieved by a smartwatch or bio-cyber device that plays the role of transducer. Once the smartwatch has processed the $\mathrm{THz}$ signal, it is in position to exchange data with a smartphone. It is important to recall that smartwatch and

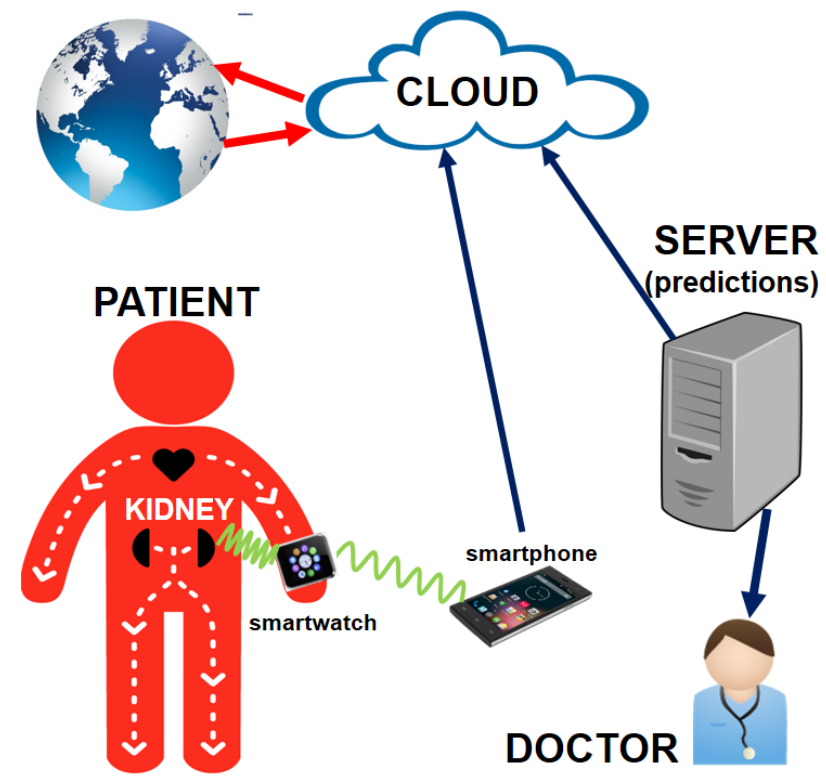

Fig. 6. Pictorial representation of the IoBNT network involving without hacking: (i) human organ, (ii) nano sensor, (iii) bio-cyber interface, (iv) smartphone, (v) cloud, (vi) world-wide-web, (vii) server, and (viii) end user. For this scheme the end user would be represented by a doctor or a health specialist. Since the doctor is continuously monitoring the possible presence of abnormalities in kidney, the requirement of an excellent QoS is needed.

smartphone already count with a IP address. We have assumed that the internet provider guarantees connectivity to the cloud in a unstoppable way. The end user, which is understood to be a health specialist (doctor or nurse, for instance) is able to download data in two ways, (i) directly, that means that all information received by the cloud can be read by the doctor [51], and (ii) indirectly, by which the data downs to a server that makes the processing of data such as to perform computational simulations and predictions in time. Indeed, cloud is connected with the world-wide-web, where any user can have access to data, if it is allowed. This scheme is advantageous in several manners. One of them is the abandon of the typical interaction between patient and doctor to be expected be done in health centers. Normally, ordinary diagnosis of the socalled diabetic nephropathy involves several interviews, lab tests and analysis, as well as preparations and cross checking previous for the diagnosis. The present scheme would reduce the diabetic nephropathy diagnosis drastically.

\section{CONCLUSION}

In this paper, we have proposed a novel scheme for the very early detection of the DKD in those type- 2 diabetes patients. We have used physics equations and through computational simulations we have estimated values which might be relevant for future implementation of these models in a full scenario of nanomedicine. The main proposal have consisted in the idea that the negative charges of the renal glomerulus are constantly depleted by the dipole moment of the glucose which is commonly in the blood of diabetes patients [51]. The depletion of these charges is seen as the very beginning of the DKD. While the negative charges of GBM are canceled, the albumin would 
escape from microvessels and exert repulsive forces onto the remaining charges located on the surface of podocytes. It has serious consequences in the deformation morphological of podocytes in time. The bunch of albumin can be also capable to break the walls of Nephrine and therefore goes to the space of urine formation. In large times, the possibility of make a full deformation of podocytes is possible since all of them have lost the capacity to reject charged proteins. According to the Monte Carlo simulations, experiments based on nanosensors can discriminate the background and measure the signal of charged bunches of albumin in up to a $70 \%$, a $15 \%$ less than the theoretical prediction. Finally, we have evaluated the possibility of extract realistic values of the efficiency of the IoBNT network when is running. In this way, we have made a crude estimated of the QoS taking into account up to 4 elements, as well as their respective sources of attenuation as well. The simulations have shown that the QoS might be ranging between $60 \%$ and $70 \%$ which might be acceptable for the objectives of a continue surveillance inside the framework of the nanomedicine.

\section{REFERENCES}

[1] Luke P. Lee, Bionano science and technology for innovative medicine, Electron Devices Meeting (IEDM), 2010 IEEE International, 6-8 Dec. 2010, San Francisco, CA, USA

[2] Tadashi Nakano, Tatsuya Suda, Yutaka Okaie, Michael J. Moore, Athanasios V. Vasilakos, Molecular Communication Among Biological Nanomachines: A Layered Architecture and Research Issues", NanoBioscience IEEE Transactions on, vol. 13, pp. 169-197, 2014, ISSN 1536-1241.

[3] T. Suda, M. Moore, T. Nakano, R. Egashira, and A. Enomoto, Exploratory research on molecular communication between nanomachines, in Proc. Genet. Evol. Comput. Conf. 2005 (GECCO 2005).

[4] S. Hiyama, Y. Moritani, T. Suda, R. Egashira, A. Enomoto, M. Moore, and T. Nakano, Molecular communication, in Proc. NSTI Nanotechnol. Conf., 2005, vol. 3, pp. 392395.

[5] S. Hiyama and Y. Moritani, Molecular communication: Harnessing biochemical materials to engineer biomimetic communication systems, Nano Commun. Netw., vol. 1, no. 1, pp. 2030, 2010.

[6] A. Goel and V. Vogel, Harnessing biological motors to engineer systems for nanoscale transport and assembly, Nature Nanotechnol., vol. 3, pp. 465475, 2008.

[7] World Health Organization, Definition, diagnosis and classification of diabetes mellitus and its complications: Report of a WHO consultation. Part 1: Diagnosis and classification of diabetes mellitus, Geneva, Switzerland, World Health Org., 1999.

[8] American Association of Clinical Endocrinologists, The American association of clinical endocrinologists medical guidelines for the management of diabetes mellitus: The AACE system of intensive diabetes selfmanagement2000 update, Endocr. Pract., vol. 6, no. 1, pp. 4384, Jan. and Feb. 2000

[9] Valeria Loscr and Anna Maria Vegni, An Acoustic Communication Technique of Nanorobot Swarms for Nanomedicine Applications, IEEE Transactions on NanoBioscience ( Volume: 14, Issue: 6, Sept. 2015 ), 598 - 607, 17 April 2015.

[10] Eisa Zarepour, Mahbub Hassan, Chun Tung Chou, Adesoji A. Adesina, Majid Ebrahimi Warkiani, Reliability analysis of time-varying wireless nanoscale sensor networks, Nanotechnology (IEEE-NANO) 2015 IEEE 15th International Conference on, pp. 63-68, 2015.

[11] Shlomi Dolev, Sergey Frenkel, Michael Rosenblit, Ram Prasadh Narayanan, K Muni Venkateswarlu, In-vivo energy harvesting nano robots, Science of Electrical Engineering (ICSEE) IEEE International Conference on the, pp. 1-5, 2016.

[12] Bryan Ronain Smith, Sanjiv Sam Gambhir, "Nanomaterials for In Vivo Imaging", Chemical Reviews, pp. , 2017, ISSN 0009-2665.

[13] Hongzhi Guo, Pedram Johari, Josep Miquel Jornet and Zhi Sun, IEEE Transactions on NanoBioscience ( Volume: 15, Issue: 1, Jan. 2016 ), 41 - 52, 11 December 2015.
[14] Burhan Gulbahar, "Theoretical Analysis of Magneto-Inductive THz Wireless Communications and Power Transfer With Multi-Layer Graphene Nano-Coils", Molecular Biological and Multi-Scale Communications IEEE Transactions on, vol. 3, pp. 60-70, 2017, ISSN 2372-2061.

[15] Mona Nafari, Josep Miquel Jornet, "Modeling and Performance Analysis of Metallic Plasmonic Nano-Antennas for Wireless Optical Communication in Nanonetworks", Access IEEE, vol. 5, pp. 6389-6398, 2017, ISSN 2169-3536.

[16] G. Piro, P. Bia, G. Boggia, D. Caratelli, L.A. Grieco, L. Mescia, "Terahertz electromagnetic field propagation in human tissues: A study on communication capabilities", Nano Communication Networks, pp. , 2016, ISSN 18787789.

[17] Gian Marco Ghiggeri, Giovanni Candiano, Gerolamo Delfino, and Carlo Queirolo, Electrical charge of serum and urinary albumin in normal and diabetic humans, Kidney International, Vol. 28 (1985), pp. 168 - 177.

[18] Akihiro Tojo and Satoshi Kinugasa, Mechanisms of Glomerular Albumin Filtration and Tubular Reabsorption, International Journal of Nephrology, Volume 2012, Article ID 481520, 9 pages doi:10.1155/2012/481520.

[19] Greiner, Walter, Classical Electrodynamics, Springer, 1998

[20] John David Jackson, Classical Electrodynamics, Third Edition 1999, John Wiley and Sons, Inc.

[21] Camelia Gabriel and Sami Gabriel, http://niremf.ifac.cnr.it/ docs/DIELECTRIC/Report.html, Physics Department King's College London, London WC2R 2LS, UK. June 1996, Final Report for the Period 15 December 1994 - 14 December 1995, Prepared for AFOSR/NL Bolling AFB DC 20332-0001.

[22] https://www.wolfram.com/mathematica/.

[23] H. Nieto-Chaupis, Prospects for anticipating kidney damage in type-2 diabetes patients through the sensing of albumin passing through the renal glomerulus, Biomedical and Health Informatics (BHI), 2017 IEEE EMBS International Conference on, 16-19 Feb. 2017, Orlando, FL, USA.

[24] Guillaume Canaud, Frank Bienaimé, Amandine Viau, Caroline Treins, William Baron, Clément Nguyen, Martine Burtin, Sophie Berissi, Konstantinos Giannakakis, Andrea Onetti Muda, Stefan Zschiedrich, Tobias B Huber, Gérard Friedlander, Christophe Legendre, Marco Pontoglio, Mario Pende and Fabiola Terzi, AKT2 is essential to maintain podocyte viability and function during chronic kidney disease, Nature Medicine 19, 12881296 (2013) doi:10.1038/nm.3313.

[25] Stuart J Shankland and Martin R Pollak, A suPAR circulating factor causes kidney disease, Nature Medicine 17, 926927 (2011) doi: $10.1038 / \mathrm{nm} .2443$.

[26] Jochen Reiser, Akt2 relaxes podocytes in chronic kidney disease, Nature Medicine 19, 12121213 (2013) doi:10.1038/nm.3357.

[27] Magzal F, Sela S, Szuchman-Sapir A, Tamir S, Michelis $\mathrm{R}$ and Kristal B (2017), In-vivo oxidized albumin a proinflammatory agent in hypoalbuminemia. PLoS ONE 12(5): e0177799. https://doi.org/10.1371/journal.pone.0177799

[28] Steven G. Coca, Girish N. Nadkarni, Yuan Huang, Dennis G. Moledina, Veena Rao, Jane Zhang, Bart Ferket, Susan T. Crowley, Linda F. Fried, and Chirag R. Parikh, Plasma Biomarkers and Kidney Function Decline in Early and Established Diabetic Kidney Disease, JASN ASN.2016101101; published ahead of print May 5, 2017.

[29] Lennart Tonneijck, Marcel H.A. Muskiet, Mark M. Smits, Erik J. van Bommel, Hiddo J.L. Heerspink, Daniel H. van Raalte, and Jaap A. Joles, Glomerular Hyperfiltration in Diabetes: Mechanisms, Clinical Significance, and Treatment, JASN April 2017 28: 1023-1039; published ahead of print January 31, 2017.

[30] Suheir Assady, Nicola Wanner, Karl L. Skorecki, and Tobias B. Huber, New Insights into Podocyte Biology in Glomerular Health and Disease, JASN ASN.2017010027; published ahead of print April 12, 2017.

[31] Pazit Beckerman and Katalin Susztak, Sweet Debate: Fructose versus Glucose in Diabetic Kidney Disease, ASN November 2014 25: 23862388; published ahead of print May 29, 2014.

[32] Christian Rask-Madsen and George L. King, Kidney complications: Factors that protect the diabetic vasculature, Nature Medicine 16, 4041 (2010) doi:10.1038/nm0110-40.

[33] Chuang PY, Dai Y, Liu R, He H, Kretzler M, Jim B, et al. (2011) Alteration of Forkhead Box O (Foxo4) Acetylation Mediates Apoptosis of Podocytes in Diabetes Mellitus. PLoS ONE 6(8): e23566. https://doi.org/10.1371/journal.pone.0023566.

[34] Wickman L, Hodgin JB, Wang SQ, Afshinnia F, Kershaw D, Wiggins RC (2016) Podocyte Depletion in Thin GBM and Alport Syndrome. PLoS ONE 11(5): e0155255. https://doi.org/10.1371/journal.pone.0155255.

[35] Fang L, Zhou Y, Cao H, Wen P, Jiang L, He W, et al. (2013) Autophagy Attenuates Diabetic Glomerular Damage through Protection of Hyperglycemia-Induced Podocyte Injury. PLoS ONE 8(4): e60546. https://doi.org/10.1371/journal.pone.0060546. 
[36] Sun YBY, Qu X, Zhang X, Caruana G, Bertram JF, Li J (2013) Glomerular Endothelial Cell Injury and Damage Precedes That of Podocytes in Adriamycin-Induced Nephropathy. PLoS ONE 8(1): e55027. https://doi.org/10.1371/journal.pone.0055027.

[37] Petrica L, Vlad A, Gluhovschi G, Gadalean F, Dumitrascu V, Gluhovschi C, et al. (2014) Proximal Tubule Dysfunction Is Associated with Podocyte Damage Biomarkers Nephrin and Vascular Endothelial Growth Factor in Type 2 Diabetes Mellitus Patients: A Cross-Sectional Study. PLoS ONE 9(11): e112538. https://doi.org/10.1371/journal.pone.0112538.

[38] Zhao L, Wu F, Jin L, Lu T, Yang L, Pan X, et al. (2014) Serum CXCL16 as a Novel Marker of Renal Injury in Type 2 Diabetes Mellitus. PLoS ONE 9(1): e87786. https://doi.org/10.1371/journal.pone.0087786.

[39] Ma J, Chadban SJ, Zhao CY, Chen X, Kwan T, Panchapakesan U, et al. (2014) TLR4 Activation Promotes Podocyte Injury and Interstitial Fibrosis in Diabetic Nephropathy. PLoS ONE 9(5): e97985. https://doi.org/10.1371/journal.pone.0097985.

[40] Hirose T, Satoh D, Kurihara H, Kusaka C, Hirose H, Akimoto K, et al. (2009) An Essential Role of the Universal Polarity Protein, aPKC, on the Maintenance of Podocyte Slit Diaphragms. PLoS ONE 4(1): e4194. https://doi.org/10.1371/journal.pone.0004194.

[41] Isaline Rowe, Marco Chiaravalli, Valeria Mannella, Valeria Ulisse, Giacomo Quilici, Monika Pema, Xuewen W Song, Hangxue Xu, Silvia Mari, Feng Qian, York Pei, Giovanna Musco and Alessandra Boletta, Defective glucose metabolism in polycystic kidney disease identifies a new therapeutic strategy, Nature Medicine 19, 488493 (2013) doi:10.1038/nm.3092.

[42] Sapna Devi, Anqi Li, Clare L V Westhorpe, Camden Y Lo, Latasha D Abeynaike, Sarah L Snelgrove, Pam Hall, Joshua D Ooi, Christopher G Sobey, A Richard Kitching and Michael J Hickey, Multiphoton imaging reveals a new leukocyte recruitment paradigm in the glomerulus, Nature Medicine 19, 107112 (2013) doi:10.1038/nm.3024.

[43] Ray Harris, EGFR signaling in podocytes at the root of glomerular disease, Nature Medicine 17, 11881189 (2011) doi:10.1038/nm.2455.

[44] Charlotte Schubert, Remodeling in the kidney, Nature Medicine 12, 391 (2006) doi:10.1038/nm0406-391.

[45] I. F. Akyildiz, M. Pierobon, S. Balasubramaniam and Y. Koucheryavy, The internet of Bio-Nano things, IEEE Communications Magazine ( Volume: 53, Issue: 3, March 2015 ), 32 - 40, 18 March 2015.

[46] Ian F. Akyildiz and Josep Miquel Jornet, The Internet of nano-things, IEEE Wireless Communications ( Volume: 17, Issue: 6, December 2010
), 1536-1284, 23 December 2010.

[47] Sasitharan Balasubramaniam and Jussi Kangasharju, Realizing the Internet of Nano Things: Challenges, Solutions, and Applications, Computer ( Volume: 46, Issue: 2, Feb. 2013 ), 62 - 68, 20 November 2012.

[48] Mahdi H. Miraz, Maaruf Ali, Peter S. Excell and Rich Picking, A review on Internet of Things (IoT), Internet of Everything (IoE) and Internet of Nano Things (IoNT), Internet Technologies and Applications (ITA), 2015, 8-11 Sept. 2015, Wrexham, UK.

[49] Josep Miquel Jornet and Ian F. Akyildiz, The internet of multimedia nano-things in the Terahertz band, Wireless Conference (European Wireless), 2012 18th European, 18-20 April 2012, Poznan, Poland.

[50] Massimiliano Pierobon, Zahmeeth Sakkaff, Jennie L. Catlett and Nicole R. Buan, Mutual information upper bound of molecular communication based on cell metabolism, Signal Processing Advances in Wireless Communications (SPAWC), 2016 IEEE 17th International Workshop on, 3-6 July 2016, Edinburgh, UK.

[51] Manish R. Maski and Samir M. Parikh, The Vasculature in Diabetic Nephropathy: All Tied Up?, JASN January 2014 25: 1-3; published ahead of print October 10, 2013.

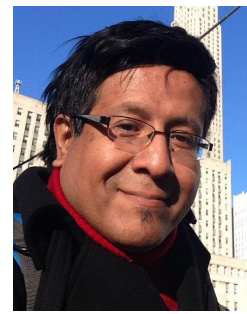

Huber Nieto-Chaupis received his Ph.D. degree in experimental physics from the MathematischNaturwissenschaftlichen Fakultät I, Humboldt Universität zu Berlin at Germany in 2007 working for the future high energy photon collider. He holds a M.Sc. in physics from the Pontificia Universidad Católica del Perú (2003), and Licenciatura in physics from the Universidad Nacional Mayor de San Marcos (1999). He was guest scientist in the Academia Sinica Taipei, Taiwan (2007), and has served as foreigner expert at the University of Science and Technology in Hefei, People Republic of China (2009). From 2010 he is an active researcher in applied physics and advanced topics of Monte Carlo simulation. He is currently researcher in the E-Health Research Center of the Universidad de Ciencias y Humanidades, Lima. 